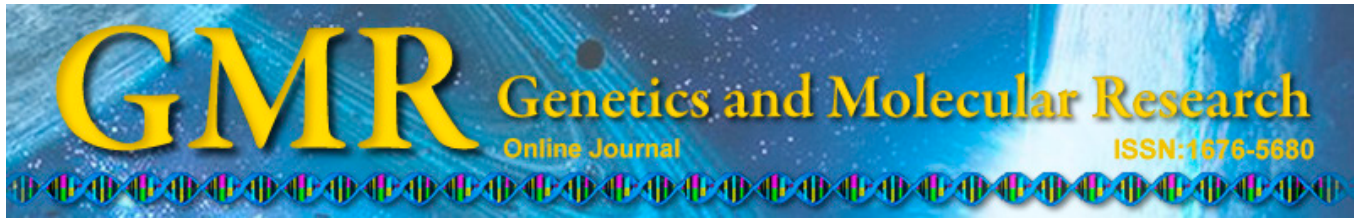

\title{
Microsatellite analysis of genetic diversity and genetic structure in five consecutive breeding generations of mandarin fish Siniperca chuatsi (Basilewsky)
}

T.L. Yi*, W.J. Guo*, X.F. Liang, M. Yang, L.Y. Lv, C.X. Tian, Y. Song, C. Zhao and J. Sun

College of Fisheries, Key Lab of Freshwater Animal Breeding, Ministry of Agriculture, Huazhong Agricultural University, Wuhan, Hubei, China Hubei Collaborative Innovation Center for Freshwater Aquaculture, Wuhan, Hubei, China

*These authors contributed equally to this study.

Corresponding author: X.F. Liang

E-mail: xufang_liang@hotmail.com

Genet. Mol. Res. 14 (1): 2600-2607 (2015)

Received March 14, 2014

Accepted July 22, 2014

Published March 30, 2015

DOI http://dx.doi.org/10.4238/2015.March.30.19

\begin{abstract}
In this report, 10 polymorphic microsatellites were applied to assess the genetic diversity and genetic differentiation of 5 consecutive breeding generations of mandarin fish, Siniperca chuatsi (Basilewsky). The results from total number of alleles, average polymorphism information content, and average homozygosity and heterozygosity showed that the genetic diversity of the breeding population was decreasing. The genetic identity between $\mathrm{F}_{1}$ and its descendant generations $\left(\mathrm{F}_{2}, \mathrm{~F}_{3}, \mathrm{~F}_{4}, \mathrm{~F}_{5}\right)$ decreased (from 0.9248 to 0.8803 ), while the genetic distance (from 0.0782 to 0.1275 ) and fixation index (from 0.03796 to 0.07393 ) increased. The allele frequency of SS181235 and SS211-246 changed regularly in the 5 breeding generations,
\end{abstract}


and they may be negatively associated with the selected trait, which needs to be confirmed by further research. Our study indicated that selective breeding was an efficient strategy for mandarin fish. In the process of breeding, some deleterious genes were phased out, and the genetic structure of the breeding populations became stable.

Key words: Mandarin fish (Siniperca chuatsi); Microsatellite; Genetic diversity; Genetic structure

\section{INTRODUCTION}

Mandarin fish, Siniperca chuatsi (Basilewsky), which belongs to Perciformes, a diverse group of fish (Nelson, 1994), is one of the chief economic freshwater fishes for aquaculture in China (Liu et al., 1998). Because of its delicious flesh, fast growth, and broad temperature tolerance range (Liang, 1996; Tao et al., 2007), mandarin fish has been cultured in many provinces of China, including Guangdong Province, where it is the pillar industry in this district.

Most cultivated mandarin fish stocks in current use in China are genetically similar or inferior to wild individuals, and no elite strains have been established so far. Moreover, the breeders of some fish farms lack of the knowledge of fish breeding, and they blindly increase the quantity of mandarin fish regardless of its quality in the process of breeding. After several generations of breeding, the genetic diversity in stocks may dramatically decline, leading to the recession of elite characteristics, such as growth, disease resistance, and suitability. To improve this situation, 5 consecutive breeding generations of mandarin fish, implemented by Hubei Collaborative Innovation Center of Freshwater Aquaculture in Guangdong Province from 2008 to 2013, were constructed to improve growth traits. When compared to unselected populations, the growth rate of the fifth generation of this mandarin fish strain was increased by $20.79 \%$ (Sun et al., 2014), and indicated the benefit of selective breeding in mandarin fish.

In terms of genetics and species evolution, a species with high genetic diversity could be a great contribution to its viability, suitability, and evolutionary potential (Ma et al., 2004). However, the genetic diversity was most likely decreased in the process of selective breeding, and it could harm the breeding program. Therefore, in addition to the genetic improvement in the trait of interest, the maintenance of relatively higher genetic diversity in the breeding population is also of importance to the selection program. In this study, variation in the genetic structure among 5 generations was investigated by 10 polymorphic microsatellite markers in order to supply theoretical references to determine the subsequent breeding strategies and also accumulate experience for fish-breeding research.

\section{MATERIAL AND METHODS}

\section{Sample collections}

To perform the selective breeding of mandarin fish, in 2008, 1800 original stocks with a relatively high level of genetic variation that were used as the base population were collected from 5 locations including 2 cultured populations and 3 wild populations. During 2008-2013, 5 consecutive breeding generations of mandarin fish were established. In the process of breeding, 2000 elites from each generation were maintained as the stocks for the next generation. 
Fin chips of 36 samples that were randomly selected from each generation were sampled and stored in ethanol until use. Total genomic DNA was extracted from ethanol-preserved fin tissues using a phenol-chloroform method (Taggart et al., 1992). DNA quality was checked by $1.2 \%$ agarose gel electrophoresis, and the concentrations were estimated using a NanoDrop 2000 spectrophotometer (Thermo Scientific, USA).

\section{Microsatellite analysis}

All of the samples were genotyped for 10 variable microsatellite loci published in previous papers (Qu et al., 2012; Huang et al., 2012; 2013; Table 1). Polymerase chain reaction (PCR) was carried out in a total volume of $12.5 \mu \mathrm{L}$, containing $50 \mathrm{ng}$ template DNA, $1.25 \mu \mathrm{L} 10 \mathrm{X}$ reaction buffer, $1 \mathrm{U}$ Taq polymerase (TaKaRa, Japan), $0.25 \mu \mathrm{L} 10 \mathrm{mM}$ dNTP, $0.5 \mu \mathrm{L} 2.5 \mu \mathrm{M}$ forward and reverse primer mixture, and water to the final volume. The cycling profiles were as follows: pre-denaturation at $94^{\circ} \mathrm{C}$ for $4 \mathrm{~min} ; 30$ cycles of $94^{\circ} \mathrm{C}$ for $30 \mathrm{~s}$, annealing at the proper temperature (Table 1) for $35 \mathrm{~s}$, and $72^{\circ} \mathrm{C}$ for $40 \mathrm{~s}$; and a final $72^{\circ} \mathrm{C}$ extension for $10 \mathrm{~min}$. PCR products were separated using an automatic capillary sequencer (ABI 3130 Genetic Analyzer, Applied Biosystems, USA) at Sangon Biotech (China). The size of alleles was scored using the GeneMapper $^{\circledR}$ software version 4.0 (Applied Biosystems) by comparison with a GenScanTM 500 ROX $^{\mathrm{TM}}$ (Applied Biosystems) internal size standard.

Table 1. Primer sequences and characteristics of 10 microsatellites of Siniperca chuatsi (Basilewsky).

\begin{tabular}{|c|c|c|c|c|c|}
\hline Locus & Primer sequence $\left(5^{\prime}-3^{\prime}\right)$ & Repeat motif & Size range (bp) & $\mathrm{Ta}\left({ }^{\circ} \mathrm{C}\right)$ & Accession No. \\
\hline $\mathrm{SC} 05$ & $\begin{array}{l}\text { TCATCTGAGGACGACTCGCT } \\
\text { AACTTAACTTCCTGCTGTCCCT }\end{array}$ & ATAG) $)_{13}$ & $174-265$ & 60 & JQ686838 \\
\hline SC09 & $\begin{array}{l}\text { ATCAGCTCAACCCCTCTGCAT } \\
\text { GCATGGATGCCAGCGTGAG }\end{array}$ & $(\mathrm{GCCTGA})_{7}$ & $199-247$ & 60 & JQ686842 \\
\hline SC49 & $\begin{array}{l}\text { CGAGTGTTTGATTTCTTCCTC } \\
\text { GTGTAAATACTGAAGGCTCG }\end{array}$ & $(\mathrm{CTC})_{10}$ & $99-106$ & 60 & JQ686880 \\
\hline SC53 & $\begin{array}{l}\text { GAAATTGAAGAAGACAAGGTGATG } \\
\text { CTGCTTTTGGCAGGAGCTAA }\end{array}$ & $(\mathrm{CA})_{19}$ & $224-276$ & 58 & JQ686884 \\
\hline $\mathrm{SC} 72$ & $\begin{array}{l}\text { CAGGGAACGGGGAAAAAGCA } \\
\text { AGAATTCCTCCGTACCTGTCTG }\end{array}$ & $(\mathrm{CA})_{17}$ & $217-261$ & 55 & JQ804731 \\
\hline SC67 & $\begin{array}{l}\text { ATCTGACACGATAAACCCTC } \\
\text { GCTGATGCTGAGGAGGAAAT }\end{array}$ & $(\mathrm{TG})_{18}$ & $147-198$ & 58 & JQ686896 \\
\hline SC83 & $\begin{array}{l}\text { TTTTAAAGACGGGGCAGCGG } \\
\text { ACCAACGTTTGGCGTAAAGC }\end{array}$ & $(\mathrm{TG})_{16}$ & $143-168$ & 60 & JQ804739 \\
\hline SS181 & $\begin{array}{l}\text { CACGAACTACTCGCCCTCAC } \\
\text { TCTGTGGGAACCGTGGTGAT }\end{array}$ & $(\mathrm{GAG})_{5}(\mathrm{GAT})_{8}$ & $124-144$ & 55 & JX294968 \\
\hline SS190 & $\begin{array}{l}\text { GTAATACTGTTGCACTTCGT } \\
\text { GTAGGCATCAAGTGAAGC }\end{array}$ & $(\mathrm{AC})_{12}$ & $169-209$ & 55 & JX294971 \\
\hline SS211 & $\begin{array}{l}\text { CAGCAGGAATTGGGATGAAA } \\
\text { CAGATGCGGCCAATACAAG }\end{array}$ & $(\mathrm{TAC})_{8}$ & $72-76$ & 55 & JX294975 \\
\hline
\end{tabular}

Ta, annealing temperature.

\section{Data analysis}

Allele numbers, effective allele numbers, polymorphism information content (PIC), and observed and expected heterozygosities were calculated by Popgene32 (Yeh et al., 2000). Genetic identity, genetic distance, and genetic differentiation between generations were assessed using Arlequin3.01 (Schneider et al., 2000). The unweighted pair-group method with 
arithmetic mean (UPGMA) phylogenetic tree was constructed with MEGA4 (Tamura et al., 2007) based on the genetic distance between generations.

\section{RESULTS}

\section{Genetic diversity of microsatellites among generations}

In this study, 10 microsatellites were used to assess the genetic diversity of the 5 consecutive breeding populations, each of which had 79, 72, 55, 66, and 64 alleles, respectively. The observed alleles were not completely consistent among generations, and a total of 110 alleles were detected in the 5 populations. Allele numbers, effective allele numbers, and observed and expected heterozygosities for each generation are summarized in Table 2. Overall, the values of these parameters declined from generation to generation except for a few parameters detected in some generations.

Table 2. Genetic diversity in selected generations of Siniperca chuatsi (Basilewsky).

\begin{tabular}{lccccc}
\hline Generation & $N_{\mathrm{E}}$ & $N_{\mathrm{A}}$ & $H_{\mathrm{O}}$ & $H_{\mathrm{E}}$ & PIC \\
\hline $\mathrm{F}_{1}$ & 3.4445 & 7.9000 & 0.7100 & 0.6565 & 0.6041 \\
$\mathrm{~F}_{2}$ & 2.8175 & 7.2000 & 0.6368 & 0.6001 & 0.5507 \\
$\mathrm{~F}_{3}$ & 2.5592 & 5.5000 & 0.5564 & 0.5508 & 0.4907 \\
$\mathrm{~F}_{4}$ & 2.7458 & 6.6000 & 0.5342 & 0.5873 & 0.5410 \\
$\mathrm{~F}_{5}$ & 2.9080 & 6.4000 & 0.4972 & 0.5959 & 0.5525 \\
\hline
\end{tabular}

$N_{\mathrm{E}}$, effective number of alleles; $N_{\mathrm{A}}$, number of alleles; $H_{\mathrm{O}}$, observed heterozygosity; $H_{\mathrm{E}}$, expected heterozygosity; PIC, polymorphism information content.

\section{Variations in allele frequency among generations}

In order to understand the genetic variation in the 5 consecutive breeding generations, the allele frequency was an important parameter that was used to estimate the level of genetic variation among generations. In this study, variation in the allele frequency could be divided into 2 classes. First, the frequency of some alleles gradually declined in the process of selective breeding, such as SS181-235 and SS211-246 (suffix number corresponding to the allele size of a locus; Figure 1). In the first generation, the 2 alleles were present at a relatively high frequency; however, with selective breeding, these alleles generally decreased and became weak alleles in the descendant generations. Second, some loci randomly changed in frequency, which fluctuated or remained nearly unchanged among various generations (data not shown).

\section{Genetic variation and differentiation among generations}

The pairwise Nei's genetic distance and genetic identity are presented in Table 3, which shows that the genetic identity between $\mathrm{F}_{1}$ and its descendant generations $\left(\mathrm{F}_{2}, \mathrm{~F}_{3}, \mathrm{~F}_{4}\right.$, and $\mathrm{F}_{5}$ ) decreased (from 0.9248 to 0.8803 ), while the genetic distance increased (from 0.0782 to 0.1275 ). The UPGMA phylogenetic tree (Figure 2) was built based on the genetic distance, which directly revealed the genetic similarities among the 5 generations of selective breeding.

Results from molecular analysis of variance (Table 4) revealed significantly larger variance within generations than among generations (96.36 and 3.64\%, respectively). The 
genetic differentiation within generations is shown in Table 5, and the total fixation index $\left(F_{\mathrm{ST}}\right)$ value was 0.03637 . The $F_{\mathrm{ST}}$ values gradually decreased between adjacent generations (from 0.03796 to 0.00968 ).

\section{a}

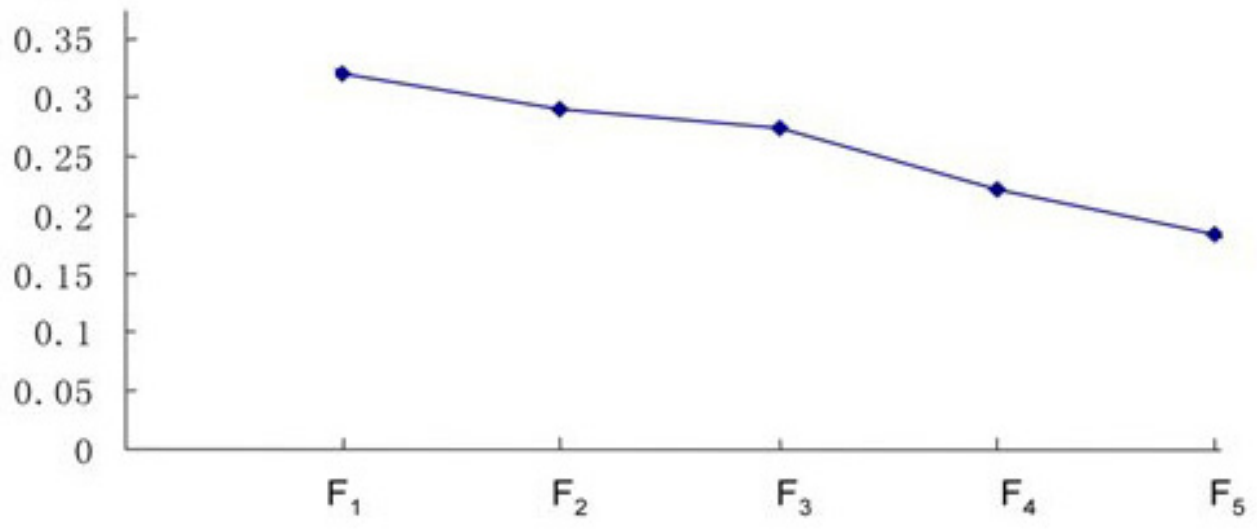

b

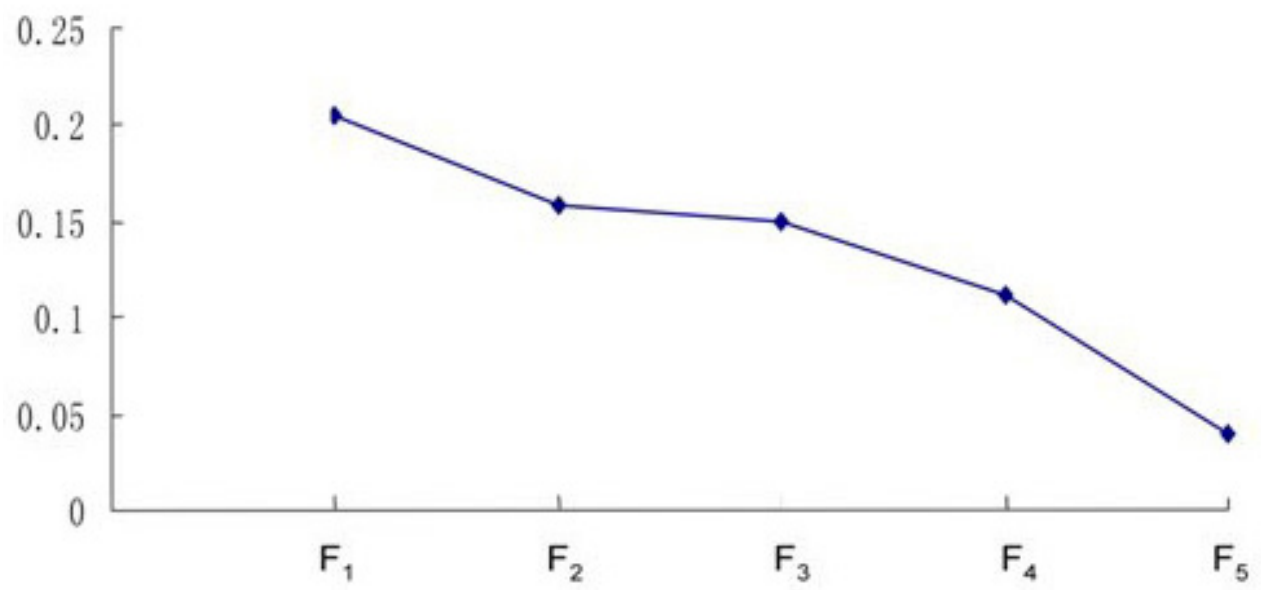

Figure 1. Allele frequency changes of SS181-235 and SS211-246 in the 5 generations. a. Allele frequency changes of SS181-235 in the 5 generations. b. Allele frequency changes of SS211-246 in the 5 generations.

Table 3. Nei's genetic distance (below diagonal) and genetic identity (above diagonal) of selected generations of Siniperca chuatsi (Basilewsky).

\begin{tabular}{lccccc}
\hline Generation & $\mathrm{F}_{1}$ & $\mathrm{~F}_{2}$ & $\mathrm{~F}_{3}$ & $\mathrm{~F}_{4}$ & $\mathrm{~F}_{5}$ \\
\hline $\mathrm{F}_{1}$ & - & 0.9248 & - & 0.9070 & 0.9008 \\
$\mathrm{~F}_{2}$ & 0.0782 & 0.0553 & 0.9462 & 0.9232 & 0.8803 \\
$\mathrm{~F}_{3}$ & 0.0976 & 0.0799 & - & 0.9570 & 0.9083 \\
$\mathrm{~F}_{4}$ & 0.1045 & 0.0961 & 0.0440 & - & 0.9605 \\
$\mathrm{~F}_{5}$ & 0.1275 & 0.0403 & 0.0345 & - \\
\hline
\end{tabular}




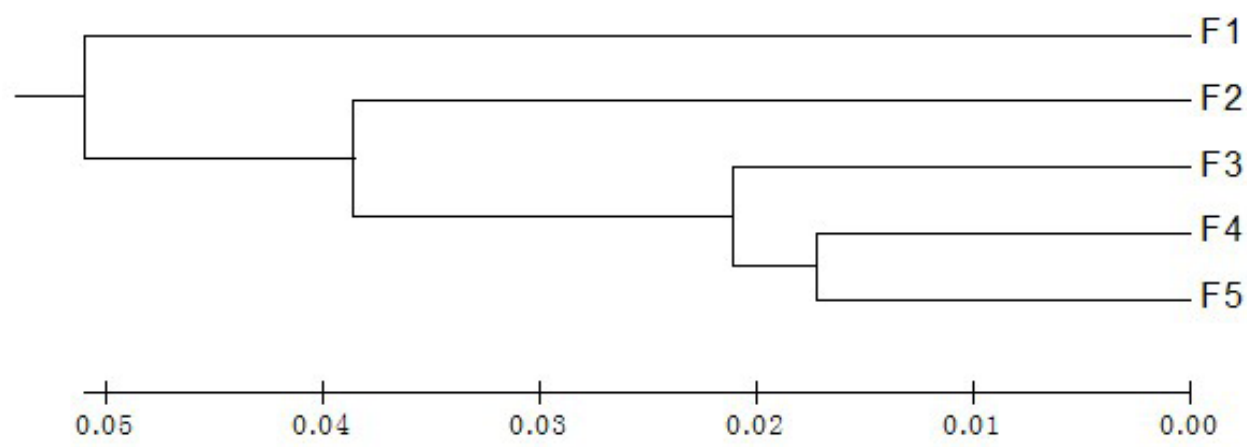

Figure 2. Unweighted pair-group method with arithmetic mean dendrogram based on Nei's genetic distance.

Table 4. Analysis of molecular variance of the 5 selective generations.

\begin{tabular}{llccr}
\hline Source of variation & d.f. & Sum of squares & Variance components & Percentage of variation \\
\hline Among generations & 4 & 46.467 & 0.11295 & 3.64 \\
Within generations & 377 & 1128.168 & 2.99249 & 96.36 \\
Total & 381 & 1174.644 & 3.10543 & 100.00 \\
\hline
\end{tabular}

Table 5. Fixation index values of pairwise comparisons among all generations.

\begin{tabular}{lccccc}
\hline Generation & $\mathrm{F}_{1}$ & $\mathrm{~F}_{2}$ & $\mathrm{~F}_{3}$ & $\mathrm{~F}_{4}$ & $\mathrm{~F}_{5}$ \\
\hline $\mathrm{F}_{1}$ & - & - & - & - & - \\
$\mathrm{F}_{2}$ & 0.03796 & - & - & - & - \\
$\mathrm{F}_{3}$ & 0.04494 & 0.2091 & 0.01452 & - & - \\
$\mathrm{F}_{4}$ & 0.05315 & 0.03766 & 0.01879 & 0.00968 & - \\
$\mathrm{F}_{5}$ & 0.07393 & 0.05400 & & - \\
\hline
\end{tabular}

\section{DISCUSSION}

Efforts have been made to build selective generations of mandarin fish from the traditional selective breeding method. Compared to unselected populations, the selected generations had obviously improved growth traits. Several studies have been performed to analyze the genetic information of consecutively selected populations of aquatic species using microsatellites aimed to improve breeding strategies (Xie et al., 2007; Zheng et al., 2007; Zhao et al., 2010); however, such a study has not yet been performed in S. chuatsi. Therefore, the aim of this study was to estimate the genetic variation and genetic structure of the strain in order to provide good advice for future breeding research.

In the first generation, the PIC ranged from 0.3941 at SC09 to 0.8705 at SS211, with an average of 0.6041 , and the number of alleles per locus varied from 3 to 19 , with a mean number of 7.9 (data not shown). According to Barker's suggestion (1994), a certain microsatellite locus that contained at least 4 alleles could be a good marker to estimate the genetic variation; this indicated that all microsatellite markers used in this study are adequate for our research.

To some extent, selective breeding may lead to decreased population genetic diversity, resulting in a homozygous population. Such an observation was also detected in 
our study and was reflected by the gradual decrease in parameter values including numbers of alleles and observed and expected average heterozygosity from $\mathrm{F}_{1}$ to $\mathrm{F}_{5}$. Several parameters among generations did not completely obey this regular change; this may be because of the small number of samples in this study. Through selective breeding, the dominant alleles of the genes relevant to a selected trait could become homozygous, which would contribute to maintaining the stability of this trait. Meanwhile, the genetic diversity of other genes, such as those related to suitability and resistance, might decrease. Therefore, it is vital for breeders to balance the homozygosity of target genes with the genetic diversity in the breeding population. In this study, the allele frequency of 10 microsatellites in the 5 generations presented 2 patterns. Type 1 (SS181-235 and SS211246) had an allele frequency that regularly changed from $\mathrm{F}_{1}$ to $\mathrm{F}_{5}$; the change tended to be negatively correlated with the growth rate. This result indicated that these alleles were more likely to be linked to deleterious genes of the growth trait. In other words, under selective stress, the deleterious alleles were gradually phased out from generation to generation, thus exhibiting a regular change in the frequency of these alleles. Further studies including association analysis should be implemented to confirm whether these 2 loci are significantly associated with the breeding trait. If the 2 loci significantly affect the breeding trait, they can be used as indicators to aid the subsequent breeding work.

The fact that heterozygotes are more fit than homozygotes has been well documented, and homozygosity could cause deleterious recessive or partially recessive lethal alleles to show their effects on the phenotype (Holtsford, 1996; He et al., 2001). Fortunately, after 5 generations, the mean observed and expected heterozygosity detected in $\mathrm{F}_{5}$ was 0.4972 and 0.5959 , respectively, indicating that the fifth generation still has high genetic diversity and could be used as stocks for selective breeding.

Artificial breeding might change the genetic structure of a population (Zhao et al., 2010). In this study, the total genetic differentiation of generations was 0.03637 , which pointed to weak differentiation according to the classification by Wright (1949). The values of genetic differentiation between adjacent generations gradually decreased, and the differentiation was weak between $\mathrm{F}_{4}$ and $\mathrm{F}_{5}\left(0<F_{\mathrm{ST}}=0.00968<0.05\right)$. At the same time, the genetic distance gradually decreased, while the genetic identity gradually increased. These results indicated that under selective stress, the genetic variation of breeding populations generally stabilized, and the tendency of this was mainly consistent with that of the growth trait. To summarize, selective breeding of mandarin fish through 5 generations, to some extent, changed the genetic structure of the breeding population and stabilized the genetic variation and the heredity of the target trait. This demonstrated that the selective breeding that we applied in mandarin fish was efficient.

The basic population in this study was composed of 3 natural populations and 2 cultured populations, which have been described as populations with high genetic diversity (Tian et al., 2013; Yan M, Tian CX, Zheng HZ, Yuan YC, et al., unpublished results). Therefore, these populations were appropriate materials to carry out the selective breeding study. After 5 generations, the genetic diversity of $\mathrm{F}_{5}$ fell about $30 \%$ relative to that of $\mathrm{F}_{1}$, indicating that $\mathrm{F}_{5}$ may also have the potential for further selective breeding. However, if blind breeding study continues, populations with low genetic diversity could lead to inbreeding depression. Therefore, the genetic diversity within each generation should be traced and monitored in subsequent breeding research. 


\section{ACKNOWLEDGMENTS}

Research supported by the National Basic Research Program of China (\#2014CB138601), the Key Projects in the National Science \& Technology Pillar Program during the Twelfth Five-year Plan Period (\#2012BAD25B04), and the National Natural Science Foundation of China (\#31272641 and \#31172420).

\section{REFERENCES}

Barker JSF (1994). A global protocol for determining genetic distances among domestic livestock breeds. In: Proceedings of the 5th world congress on genetics applied to livestock production. University of Guelph, 21: 501-508.

He TH, Rao GY, You RL, Ge S, et al. (2001). Genetic structure and heterozygosity variation between generations of Ophiopogon xylorrhizus (Liliaceae sl), an endemic species in Yunnan, southwest China. Biochem. Genet. 39: 93-98.

Holtsford TP (1996). Variation in inbreeding depression among families and populations of Clarkia tembloriensis (Onagraceae). Heredity 76: 83-91.

Huang W, Liang XF, Qu CM, Li J, et al. (2012). Isolation and characterization of twenty-five polymorphic microsatellite markers in Siniperca scherzeri Steindachner and cross-species amplification. J. Genet. 91: e113-e117.

Huang W, Liang XF, Qu CM, Cao JJ, et al. (2013). Development and characterization of novel polymorphic microsatellite loci in Siniperca scherzeri Steindachner and Siniperca chuatsi (Basilewsky). Mol. Biol. Rep. 40: 751-756.

Liang XF (1996). Study on mandarin fish and its culture home and abroad. Fish. Sci. Technol. Inform. 23: 13-17.

Liu J, Cui Y and Liu J (1998). Food consumption and growth of two piscivorous fishes, the mandarin fish and the Chinese snakehead. J. Fish Biol. 53: 1071-1083.

Ma C, Liu M, Ma L, Zhang F, et al. (2004). Genetic diversity in Coilia ectenes by RAPD analysis. Mar. Fish. Res. 25: $19-24$.

Nelson JS (1994). Fishes of the World. John Wiley and Sons, New York.

Qu CM, Liang XF, Huang W, Zhao C, et al. (2012). Development and characterization of twenty-nine novel polymorphic microsatellite loci in the mandarin fish Siniperca chuatsi. J. Genet. 92: e19-e23.

Schneider S, Roessli D and Excoffier L (2000). Arlequin: A Software for Population Genetics data Analysis. University of Geneva, Geneva, 2496-2497.

Sun LF, Li J, Liang XF, Yi TL, et al. (2014). Evaluation of selective breeding effect of Siniperca chuatsi. Guangdong Agr. Sci. 41: 114-118.

Taggart JB, Hynes RA, Prodöuhl PA and Ferguson A (1992). A simplified protocol for routine total DNA isolation from salmonid fishes. J. Fish Biol. 40: 963-965.

Tamura K, Dudley J, Nei M and Kumar S (2007). MEGA4: molecular evolutionary genetics analysis (MEGA) software version 4.0. Mol. Biol. Evol. 24: 1596-1599.

Tao JJ, Gui JF and Zhang QY (2007). Isolation and characterization of a rhabdovirus from co-infection of two viruses in mandarin fish. Aquaculture 262: 1-9.

Tian CX, Yang M, Liang XF, Cao L, et al. (2013). Population genetic structure of Siniperca chuatsi in the middle reach of the Yangtze River inferred from mitochondrial DNA and microsatellite loci. Mitochondrial DNA 0: 1-7.

Wright S (1949). The genetical structure of populations. Ann. Eugenic. 15: 323-354.

Xie XY, Li SF and Cai WQ (2007). Analysis of genetic diversity of GIFT strain Nile tilapia (Oreochromis niloticus) during selection processing by microsatellites. J. Fish. China 31: 385-290.

Yeh FC, Yang R, Boyle TJ, Ye Z, et al. (2000). POPGENE 32, Microsoft Windows-based freeware for population genetic analysis. Molecular Biology and Biotechnology Centre, University of Alberta, Edmonton.

Zhao GT, Liu XD, Wang ZY, Cai MY, et al. (2010). Genetic structure and genetic diversity analysis of four consecutive breeding generations of large yellow croaker (Pseudosciaena crocea) using microsatellite markers. J. Fish. China 34: 500-507.

Zheng K, Lin KD, Liu ZH and Luo C (2007). Comparative microsatellite analysis of grass carp genomes of two gynogenetic groups and the Xiangjiang River group. J. Genet. Genomics 34: 321-330. 